\title{
Revisión crítico-bibliográfica en torno a la marca fig. en diccionarios de uso del español*
}

\author{
Constanza Martínez Gajardo** \\ Universidad de Chile
}

\section{PRESENTACIÓN Y MARCO}

En la presente nota nos proponemos revisar críticamente algunos de los textos que se han escrito en torno a la marca fig., presente en diccionarios monolingües de uso del español. Su interés se funda en el hecho de que esta marca ha sido discutida, en relación con el tipo de información que entrega respecto de la acepción y el vínculo que esta establece con el sentido recto con que forma unidad polisémica, así como por lo que refiere a los casos en que corresponde su uso en los diccionarios.

El marco en que se inscribe nuestra concepción del diccionario es el desarrollado por Luis Fernando Lara (1990) en su Teoría del diccionario monolingüe. En este sentido, adherimos a lo que Lara ha definido como la especificidad del objeto diccionario, en cuanto se trata de un texto escrito - producto de una cultura interesada por fijar y conservar un discurso que le es propio- que cumple una función específica dentro de

* Este trabajo fue escrito en el marco del curso de Semántica, dictado por el profesor Alfredo Matus Olivier, para el Magíster en Lingüística con mención en lengua española de la Universidad de Chile.

** Para correspondencia, dirigirse a: Constanza Martínez Gajardo (constanza.martinez.g@, gmail.com), Universidad de Chile, Facultad de Filosofía y Humanidades, Departamento de Filosofía, Avda. Capitán Ignacio Carrera Pinto 1025, Ñuñoa, Santiago, Chile. 
esa institucionalidad. Este discurso es el léxico, que entra en contacto directo con la realidad, nombrándola e interpretándola desde la experiencia particular de la comunidad lingüística a la que corresponde. Desde esta perspectiva, entendemos el objeto diccionario como depositario de la

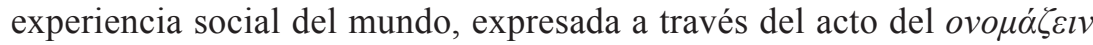
(onomázein). Así, el diccionario de uso encuentra su razón de ser en una sociedad con un alto grado de desarrollo, en la que la fragmentación del conocimiento pone en riesgo la cohesión intracomunitaria que permite el acto mismo de la comunicación efectiva y el alcance de acuerdos. El rol de este depósito físico de memoria lingüística es entonces eminentemente social: se erige como elemento de cohesión que asegura el entendimiento de una comunidad cuyo conocimiento desborda la capacidad individual de contenerlo. Este hecho, natural al desarrollo de cualquier lengua, representa su mismo carácter social y hace patente la reflexión de sus hablantes sobre ella. El diccionario es entonces una institución social, es decir, un depósito comunitario de la memoria de experiencias individuales que aspira a cumplir la función de asegurar el consenso con respecto a los sentidos que se construyen desde la lengua, más allá de la fugacidad del discurso en el presente.

Por otra parte, y aún siguiendo a Lara, el diccionario es -como toda expresión verbal-, un acto de habla y, en cuanto tal, está dotado de un sentido social al interior del entramado de todos los actos de lenguaje. Su valor o función puede describirse así como el de un "catálogo codificado de actos verbales de respuesta" (1990:34) frente a la pregunta por el significado de las unidades léxicas de una lengua, formulada por un individuo perteneciente a la comunidad lingüística a la que dicho conocimiento pertenece, donde el verbo "significa", elidido entre el lema y la definición, establece una relación de sinonimia entre ambos elementos. El valor de verdad con que esta respuesta sea aceptada deriva de la institucionalidad misma del diccionario: de su capacidad de ser continente de la memoria, no de un grupo minoritario de hablantes, sino de la comunidad en sí.

De todo lo expuesto se deduce que el ideal de un diccionario semasiológico monolingüe de uso es que establezca su nomenclatura sustentándola en corpora reales de lengua en uso, que busquen ofrecer una mirada descriptiva, antes que normativa, capaz de dar cuenta de lo que Coseriu (1977) describió como la arquitectura de la lengua. Esta última corresponde al conjunto de relaciones que implica la multiplicidad de las técnicas del discurso coexistentes en la lengua histórica, esto es, del conjunto de dialectos, niveles y estilos de lengua que coexisten, ya no solo en una comunidad lingüística, sino en cada uno de sus hablantes. 


\section{MÉTODOS DE APROXIMACIÓN AL PROBLEMA}

Dado que el propósito de esta nota es solo realizar una revisión crítica de los textos que tratan sobre la marca fig. en diccionarios de uso del español, será pertinente revisar, a través de la literatura, algunos elementos generales del sistema de marcación del diccionario y del tipo de información que entrega respecto a la unidad léxica (en adelante, U.L.), centrándonos en la marca fig. A continuación, revisaremos en la bibliografía la historia de esta marca en el Diccionario de la Real Academia Española (DRAE) y también las lecturas que, desde el estudio directo de sus aplicaciones, algunos autores han hecho acerca del tipo de información que entrega dicha marca en ese diccionario. Al mismo tiempo, revisaremos, brevemente, cómo se comporta esta marca - cuando se presenta- en otros de los principales diccionarios de uso del español, como son el Diccionario del Español Actual (DEA) y el Diccionario de Uso del Español (DUE), a través de algunos ejemplos que hemos podido recoger directamente.

En torno al tratamiento de la polisemia léxica en el diccionario, será relevante acercarnos a algunas de las visiones teórico-lexicográficas que están vigentes en la actualidad para revisar sus postulados, respecto del tipo de relación que se establece entre sentido recto y figurado de una U.L. polisémica. Finalmente, a la luz de lo anterior y de nuestra concepción de diccionario, intentaremos comprender cuál es la visión respecto a la polisemia léxica por transición semántica metafórica, que expresa la Academia a través de la supresión de la marca fig. en la edición de 2001, a fin de intentar dilucidar cuál sería un adecuado tratamiento lexicográfico, desde nuestra perspectiva, para los sentidos figurados en el diccionario de uso monolingüe y descriptivo, al cual adscribimos.

\section{LA MARCA FIG. DENTRO DEL SISTEMA DE MARCACIÓN EN EL DICCIONARIO DE USO DEL ESPAÑOL}

\subsection{LA MARCA FIG. SEGÚN FAJARDO}

Para revisar algunos aspectos de la marca fig. dentro del sistema de marcación en la microestructura del artículo del diccionario semasilógico, nos remitiremos a Fajardo (1996-1997), quien realiza una revisión crítica del uso de los sistemas de marcas que ha hecho la lexicografía española. 
Desde la perspectiva de Fajardo, "Marcación es el recurso o procedimiento que se utiliza en el diccionario para señalar la particularidad de uso, de carácter no regular, que distingue a determinados elementos léxicos. Su valor es general y afecta a todo el diccionario, de manera que las unidades léxicas quedan divididas en marcadas y no marcadas" (1996-1997:31-32).

Para Fajardo, la función principal de la marcación en el diccionario es caracterizar a un elemento léxico, señalando sus restricciones y condiciones de uso. Se expresa, idealmente, a través de marcas sistemáticas que entregan al usuario informaciones concretas sobre dichas condiciones y restricciones. Por lo regular, el sistema de marcas consiste, en términos de presentación gráfica, en un conjunto de abreviaturas que suelen estar tras el lema y antes de la definición. Sin embargo, es claro que ni toda marca es una abreviatura, ni toda abreviatura en el diccionario es una marca. De hecho, Fajardo revisa críticamente este punto, determinando que muchas de las llamadas marcas, en realidad son solo indicaciones complementarias, que comprenden un "conjunto de informaciones que aparecen en el artículo lexicográfico con distintos grados de formalización, pero que no se refieren directamente a la marcación lingüística de la unidad léxica" (1996-1997:32).

En este sentido, Fajardo considera que las marcas, en sentido estricto, son solo aquellas que entregan información respecto de la U.L. de los siguientes tipos: diacrónica, diatópica, de nivel, registro y estilo, técnicas, de tipo connotativo, de frecuencia de uso y normativas. Sin duda, cada uno de estos grupos ofrece, mirados y contrastados sus usos en los diccionarios del español, aspectos problemáticos, dado que: a) algunas marcas son interpretadas de manera distinta por unos y otros diccionarios, como ocurre con fam. y col., b) otras ofrecen una gradación subjetiva, como las de frecuencia de uso y c) algunas implican un alto grado de juicio valorativo por parte del equipo lexicográfico, como ocurre con marcas de tipo connotativo que sirven para indicar la intención despectiva, festiva o irónica del hablante. Lo relevante para este trabajo, sin embargo, es el hecho de que Fajardo deja fuera del sistema de marcas aquellas abreviaturas que contienen información con respecto a la categoría gramatical, los préstamos lingüísticos, las indicaciones contrastivas propias de los diccionarios diferenciales y las indicaciones complementarias respecto de transiciones semánticas o categoriales; puesto que no considera que expresen restricciones o condiciones de uso de la U.L. Con ello, para Fajardo, fig. no sería una marca, sino solamente una indicación complementaria expresada a través de una observación abreviada regularmente de acuerdo con una fórmula fija. Expuesto esto, Fajardo no profundiza respecto del tipo de información que la abreviatura entrega. Creemos que la valoración del autor al respecto es errada, ya que, como intentaremos demostrar a través de este trabajo, resulta contradictorio señalar 
que la abreviatura fig. es solo una indicación complementaria y al mismo tiempo atribuir a las marcas la función de caracterizar a la U.L., señalando sus restricciones y condiciones de uso.

\subsection{La MarCa Fig. SEgún SeCo}

En nuestro análisis de la clasificación general del tipo de información a la que pertenece la entregada por la abreviatura fig., revisaremos el artículo clásico de Manuel Seco, "Problemas formales de la definición" (1978), en que se establecen dos grandes bloques de información dentro del artículo lexicográfico: la de primer y la de segundo enunciado. Para Seco, la información de primer enunciado es la que entrega información referida a la U.L. en cuanto signo, mientras que la de segundo enunciado está referida a su contenido. A su entender, entonces, en el primer bloque se integrarían noticias más bien dispares, como la etimología y la categoría gramatical de la voz, su época de vigencia, sus límites geográficos, el campo del saber o actividad a que está confinada, sus niveles de uso (diastrática y diafásicamente), sus particularidades de "colocación" respecto de contextos reales de uso o locuciones, $\mathrm{y}$-en acepciones secundarias- la explicación de transiciones semánticas y categoriales. La última categoría de información de primer enunciado mencionada por el autor es la siguiente: "f) explicación de las transiciones semánticas, por medio de abreviaturas como fig., por ext., irón. (esta información lógicamente, solo en acepciones secundarias)" (Seco 1978:16). En este punto cabe destacar dos aspectos: el primero, referido al hecho de incluir esta información dentro del primer enunciado, y el segundo, en torno de la inclusión de la abreviatura irón. dentro del grupo de las que informan sobre transición semántica. En el primer sentido, las transiciones semánticas que generan polisemia, es decir, cuya segunda acepción está gramaticalizada, nos parecen ser no un aspecto de la U.L. en cuanto signo, sino parte de lo que Seco llama el contenido de la U.L., puesto que afectan directamente a su significado. Como veremos más adelante, en términos semánticos, al menos algunos nombres utilizados en sentido figurado podrían interpretarse no con significado denotativo, sino como caracterizadores de la entidad sobre la que se predican. Por lo que se refiere al segundo aspecto, cabría mencionar que la abreviatura irón. ofrece a lo menos dos interpretaciones: a) entregando información relacionada con un proceso de derivación semántica $\mathrm{y}$, más modernamente, $\mathrm{b}$ ) informando sobre la intención del hablante al utilizar determinada U.L. En este último sentido, cabría más bien dentro de lo que Fajardo (1996-1997) llamaría marcas de tipo connotativo. 


\section{ACERCA DEL TIPO DE INFORMACIÓN QUE ENTREGA LA MARCA $F I G$.}

En relación con la abreviatura fig., de Hoyos (1999-2000) realiza un interesante estudio diacrónico en que se propone analizar en la tradición lexicográfica española "cómo los diccionarios han tratado el grupo léxico caracterizado por la marca de transición semántica sentido figurado"(p.73). Dentro de su primer acercamiento metalexicográfico, el autor señala la necesidad de revisar el valor que encierra la inclusión de descripciones lingüísticas en los artículos de un diccionario de uso y, en particular, con qué objetivo se utiliza la marca de transición semántica figurado. Pese a seguir a Fajardo (1996-1997) por lo que refiere a la función caracterizadora de las marcas, este autor se atiene a la tradición lexicográfica al considerar figurado como marca. En tanto tal, señala que la abreviatura fig. aporta información adicional respecto de la acepción, integrándola bajo el principio de economía de espacio, pese a lo cual, su uso poco coherente y asistemático al interior de los diccionarios reduce las ventajas de esa aportación de sentido.

Para revisar hasta qué punto los principios de sistematicidad y coherencia, bajo los cuales se estructuran idealmente los diccionarios, se cumplen o no respecto de esta marca, el autor analiza los procedimientos por los que se entrega este tipo de información a partir del mismo Tesoro de la lengua castellana o española de Covarrubias. A través de este procedimiento -el estudio directo de la aplicación de la marca sobre un corpus acotado (zoónimos)-, de Hoyos pretende, a su vez, inferir la teoría académica respecto de la marca figurado, acerca de la cual critica que la Academia misma nunca ha realizado alguna aclaración. Así, llega a concluir que para su corpus, mientras el Tesoro no incluye ningún procedimiento de marcación para la acepción figurada de 'cochino', por ejemplo, la primera obra de la Academia - el Diccionario de Autoridades (DAut)- ofrece ya con cierta sistematicidad el procedimiento de incluir un descriptor semántico. Así, el autor encuentra en el DAut (1726-1739) los siguientes descriptores para su corpus: metafóricamente (con la mayor frecuencia de uso), figuradamente, traslaticiamente, por semejanza $\mathrm{y}$, con una frecuencia de uso considerablemente menor, por analogía, por traslación, por alusión y alusivamente. Siguiendo con la revisión diacrónica del uso de estos procedimientos, el autor demuestra que la Academia continuó utilizándolos con relativa sistematicidad hasta que, en el DRAE de 1780, por razones de economía de espacio, introduce la marca met. con el valor de 'metáfora o metafóricamente', la que ya en 
1884 llega a estabilizarse en su uso con la abreviatura fig., con el valor de 'figurado o figurada', de modo contundentemente sistemático. De acuerdo con de Hoyos (1999-2000: 78):

Se pasa de una marcación impresionista símbolo o significa también (Cov 1611) a una descripción lingüística precisa, que evoluciona desde el descriptor completo metafóricamente (DAUT 1726-1739) hasta el productivo fig. abreviado (DRAE 1884).

camaleón es el camaleón símbolo del hombre astuto, disimulado y sagaz, que fácilmente se acomoda al gosto y parecer de la persona con quien trata para engañarla. Significa también el lisonjero y adulador, que si lloráis llora, y si reís ríe, y si a medio día claro decís vos que es de noche, os dirá que es así, porque ve las estrellas' (Cov 1611).

camaleón 'metafóricamente se llama el adulador, que viste sus pensamientos del color de la fortuna ajena, para hacer la suya propia' (DAUT 1726-1739).

camaleón 'figuradamente se llama así al hombre que, a impulsos del favor o del interés, muda con facilidad de pareceres o doctrinas'(DRAE 1869).

camaleón..fig. y fam. (DRAE 1884).

La existencia de ocho descriptores distintos en el DAut obliga a preguntarse si se trata de una falta de sistematicidad en la marcación de un mismo fenómeno, o bien de interpretaciones diferentes para una serie de fenómenos de transición semántica percibidos como disímiles. Lo cierto es que, con la sistematización de la abreviatura fig. en 1884, podemos inferir que, al menos a partir de ese momento, la Academia unifica su percepción del fenómeno de la transición semántica metafórica.

Lo que es común a todos estos tratamientos es la concepción básica académica respecto de que existe una polisemia, fruto de la existencia de una U.L. denotativa en su acepción primaria, desde la que, por procedimientos generalmente analógicos, se deriva una acepción secundaria que se aleja del sentido recto: de ello se deriva el juego de oposición binaria 'sentido propio o recto/sentido figurado o no propio', que relaciona esta marca tanto con la tradición retórica, como con la etimológica. A su vez, esta oposición presenta su correlato respecto del orden de las acepciones, quedando la figurativa siempre subordinada a la recta. Esta oposición binaria, en última instancia, otorga un valor por negación a la marca fig., esto es, el valor de ser una acepción distinta de la recta.

En las ediciones posteriores a la de 1884, sin embargo, de Hoyos confirma cómo la sistematicidad de aplicación de la marca académica comienza a decaer. En el DRAE de 1992, por ejemplo, aparece 17.161 
veces la abreviatura fig.; 37, figs.; 5, sent.fig.; 7, Ú. en sent.fig.; 198, Ú.t.en sent.fig.; 5, Ú.m. en sent.fig. y 1, Ú.m. en frs.figuradas. Eso, sin considerar las ocasiones en que el hecho lingüístico de la figuración se expresa dentro del cuerpo de la definición a través de la palabra 'figurado'. Por ello, el autor considera que, siendo uno el concepto académico sobre la figuración, una debería también ser la marca, si se quiere participar de los principios de sistematicidad y coherencia, a los que ya nos hemos referido. Por otra parte, algunas de las voces que estaban marcadas en las ediciones anteriores como figuradas, ahora aparecen sin marca, aunque como acepciones distintas en distribución polisémica. Es el caso de la voz 'zorra', por ejemplo, que en su cuarta acepción, 'persona astuta', todavía aparece marcada como fig. en el DRAE 1970, pero ya no lo está en el de 1992. Para de Hoyos, este fenómeno es simplemente un problema de inconsistencia que termina por generar una serie de ataques respecto a los criterios lexicográficos de la Academia. De hecho, en sus conclusiones, el autor expresa que además de los problemas de coherencia y sistemacidad observados en la aplicación de la marca, "el carácter histórico y retórico de la descripción sentido figurado no permite una buena adaptación a la técnica lexicográfica" (de Hoyos 1999-2000:102), confirmando así el ambiente crítico al que algunos adjudican la desaparición de la marca en la $22^{\mathrm{a}}$ edición del diccionario, al año siguiente de la publicación de su trabajo.

Nosotros pensamos, sin embargo, que esta asistematicidad en el uso de la marca puede deberse a dos cosas: la posibilidad de que la Academia considerara dicha acepción como demasiado gramaticalizada para suscitar en el hablante la imagen o los rasgos del referente 'zorra' en sentido recto, o bien, que, como ya expresamos, la discusión con respecto al valor y la validez de esta marca -que terminará con su eliminación del diccionario en la edición de 2001- ya estuviera generando al interior de la Academia los primeros cambios, aun asistemáticos.

Analizando justamente la decisión académica de suprimir la marca fig. o, en algunos casos, cambiarla por la marca de estilo o registro coloq., Borràs y Torner (2006) escriben un artículo que, a través del estudio de un pequeño corpus de zoónimos en el DRAE 1992, busca probar que la marca fig. no es utilizada realmente en forma asistemática por la Academia, sino que podría estar enfocada solo a aquellas derivaciones semánticas que funcionan como epítetos, esto es, que pierden el carácter denotativo de la acepción primaria $\mathrm{y}$, tomando algunos rasgos del ente denotado, se prefiguran con un valor próximo al del adjetivo calificativo, admitiendo incluso modificaciones de grado. Veamos un ejemplo: 
1.a. José tiene un corral lleno de cerdos.

2.a. José es un cerdo.
1.b.*José tiene un corral lleno de muy cerdos.

2.b. José es muy cerdo.

Como puede verse, en 1.a, el sentido de la U.L. 'cerdo' es puramente denotativo, su significado hace referencia a un ente. En cambio, en 2.a y 2.b., encontramos la misma U.L. como predicación, con un uso valorativo cuyo significado destaca algunos rasgos del ente denotado por la acepción en sentido recto, lo que permite el uso del intensificador en 2.b, que resulta agramatical en 1.b. Desde este punto de vista, pueden abrirse varias implicaciones interesantes. Por una parte, cabe destacar que este fenómeno no atañe a todas las formas derivadas que pueden predicarse de personas. Esto queda demostrado en la voz 'camello', cuya segunda acepción, 'persona que vende drogas tóxicas al por menor', no cumple la función de caracterizador, sino solo la de denotar un ente, por lo que no es susceptible de utilizarse con un intensificador:

3.a. José viajó en camello. 3.b. José es mi camello. 3.c. *José es muy camello.

Además de ello, nos parece que no es evidente a la conciencia del hablante cuál es la analogía y qué rasgos compartirían el ente denotado en la acepción primaria y el denotado en la secundaria. Lo importante, en todo caso, es destacar que el DRAE de 1992 no marca la segunda acepción de 'camello' con la marca fig., lo que confirmaría, al menos para los casos estudiados por estos autores, la lectura de que fig. no es una marca puramente etimológica, sino también una marca de uso:

En este sentido, la marca fig. tal como se emplea en el DRAE (1992) no es en rigor una marca diacrónica, sino que se trata de una marca que indica el modo como debe ser leída la definición o, en otros términos, el modo como se emplea la palabra en esa acepción (Borràs y Torner 2006: 37-38).

Volviendo al ejemplo de la voz 'cerdo', por otra parte, y siguiendo en este aspecto los aportes de la semántica léxica cognitiva, podemos apreciar que una y otra acepción se 'contaminan' mutuamente, en el sentido de que la figura retórica "va y vuelve": genera una animalización del sujeto del que se predica la segunda acepción, pero también una personificación del animal denotado por la primera. A su vez, el carácter despectivo del término 'cerdo' en su acepción figurativa, no proviene exclusivamente de los rasgos que le son propios al ente connotado por la acepción primaria, sino que se sustenta en un supuesto cultural, que marca el animal connotado como símbolo de impureza, incluso desde las leyes mosaicas. En este sentido, concordamos 
con los autores en que la marca fig. es "además de semántica y de uso (uso predicativo), una marca cultural, lo cual supera las fronteras de la información meramente diacrónica o etimológica" (Borràs y Torner 2006:38). En este sentido, el cognitivismo considera que cada ítem léxico polisémico establece una red semántica que se estructura como una categoría radial y que "los contenidos constitutivos de una red semántica polisémica no se generan por efecto del contexto, sino que se encuentran instanciados en la memoria semántica de largo plazo (= "léxico mental"), es decir, los significados que la constituyen están convencionalizados" (Rojas 2010:17).

Por último, y cumpliendo con los ejes programáticos planteados, cabe mencionar que la decisión de eliminar la marca fig. no solo atañe al DRAE 2001; lo mismo ocurrió con el DUE, de María Moliner, cuya edición de 1999 eliminó por completo la marca, aun cuando, por supuesto, sigue registrando las dos o más acepciones en las unidades polisémicas producto de transición semántica, subordinando la acepción más reciente, a la que etimológicamente se considera primaria, en lo que respecta a la ordenación de las acepciones. Los motivos de la eliminación de la marca tampoco quedan expresados en la presentación del nuevo DUE. En cambio, el DEA de Manuel Seco, que vio la luz el mismo año (1999) tras treinta años de recopilación de corpus y trabajo lexicográfico, presenta la abreviatura fig., pero no la utiliza propiamente como marca de acepción figurada, sino como indicación complementaria al interior de una acepción, bajo la forma $T b$ fig., que indicaría, a nuestro entender, que en esa acepción de la U.L., además de en sentido recto, es posible utilizarla en sentido figurado. Veamos algunos ejemplos:

corazón. (...) 6. Parte central [de una cosa] Tb fig.

abajar. tr. 1 (lit) Bajar. Tb fig. (...) b) pr.Bajarse. Tb fig. (DEA 1999).

En cambio, las acepciones que tenderíamos a pensar que podrían estar marcadas como figuradas, aparecen con la marca de estilo o registro '(col): coloquial'. Así:

bufar.2. (col) Manifestar [alguien] gran enojo.

búho.2. (col) Autobús nocturno.

buitre.2. (col) Pers. codiciosa y con pocos escrúpulos (DEA 1999).

Si bien estos tres diccionarios tienen particularidades que hacen valiosísimo a cada uno de ellos (catálogo en el caso del DUE, corpus y contorno en el del DEA, y tradición, prestigio y corpus en el del DRAE), no es difícil imaginar que el mercado para los diccionarios monolingües de uso de español no es tan grande como para que la competencia no sea 
fuerte. Así, con la publicación del DUE y el DEA en 1999 -más la presión ejercida desde la lexicografía teórica respecto de la ambigüedad del uso de la marca, de la impertinencia de una marca supuestamente etimológica en un diccionario sincrónico de uso y de la asistematicidad de su aplicación, sumado todo ello a las presiones editoriales-, el DRAE se habrá visto forzado a tomar decisiones sobre el uso de la marca fig. que, como ya hemos mencionado, estaba presente en más de 17.000 acepciones en la edición anterior al 2001.

Lo que sabemos con certeza es que, en la vigésimo segunda edición del DRAE, la marca solo ha quedado en algo más de 190 voces, siempre bajo la fórmula "Ú.t. en sent fig.", que indicaría, como en el caso del DEA, que en esa acepción de la U.L., además de en sentido recto, es posible utilizarla en sentido figurado. Ejemplifiquemos:

anteojera. 2. f. pl. En las guarniciones de las caballerías de tiro, piezas de vaqueta que caen junto a los ojos del animal, para que no vean por los lados, sino de frente. Apl. a pers., u. t. en sent. fig.

templar. (Del lat. temperāre). 1. tr. Moderar, entibiar o suavizar la fuerza de algo. Apl. al genio o enojo de una persona, u. t. en sent. fig.

ajustar. (De $a^{-1}$ y el lat. iustus, justo). 2. tr. Conformar, acomodar algo a otra cosa, de suerte que no haya discrepancia entre ellas. U. t. en sent. fig. (DRAE 2001).

De estas tres voces, cabe mencionar que, en el DRAE de 1992, ya todas presentaban la indicación de uso Ú.t. en sent.fig., aun cuando el contorno se ponía tras esta información complementaria y no delante, como en la actualidad. En cambio, muchas de las que aparecían con la marca fig. en DRAE 1992 y versiones anteriores, aparecen ahora con la marca coloq. (coloquial).

cerdo. (...). 3. m. coloq. puerco (// hombre sucio). U. t. c. adj.

4. m. coloq. puerco (// hombre grosero). U. t. c. adj.

5. m. coloq. puerco (// hombre ruin). U. t. c. adj. (DRAE 2001).

Recapitulando lo que hemos expuesto, la marca fig. ha sido descrita en la literatura especializada como una marca de transición semántica que entrega información diacrónica o etimológica (bajo la oposición primaria/derivada), de tipo estilística para otros (bajo la oposición sentido recto/sentido figurado) y de indicación de uso predicativo para los últimos (bajo la oposición uso extensional o denotativo/ uso intensional o predicativo). 


\section{RELACIONES QUE SE ESTABLECEN ENTRE ACEPCIONES DE UNA POLISEMIA}

El trabajo de Borràs y Torner (2006) finaliza con el siguiente planteamiento:

“(...) no creemos que ni los errores observados en la praxis ni las dificultades inherentes que supone la marcación de los sentidos figurados justifiquen sin más su supresión. De otro modo, el diccionario no puede reflejar adecuadamente las relaciones que las entradas léxicas mantienen entre sí, que contemplan necesariamente la metáfora sincrónica ${ }^{1}$ en el sentido propuesto por van der Meer" (p. 41).

$\mathrm{Al}$ respecto, nos parece que el tema de la relación que se establece entre las distintas acepciones de una U.L. polisémica es central para comprender las actitudes que genera la marca de sentido figurado entre representantes de cada paradigma o escuela lingüística. Así, mientras para Borràs y Torner (2006) -a quienes identificamos como cercanos al enfoque cognitivista de la polisemia-algo relevante deja de expresarse con la supresión de la marca fig., para otros autores, como Pérez Castro (2002) - que parece adherir a la escuela estructuralista norteamericana-, la marca es completamente innecesaria, siendo incluso partidario de dar al artículo una estructura monosémica, puesto que, desde su perspectiva, las acepciones de sentido figurado expresan solo el "significado efectivo" de las unidades léxicas, donde "el "significado efectivo' de una locución es lo que ésta da a entender, lo que sugiere"( 128). Para Pérez Castro: “(...) los usos y sentidos figurados ni son nunca gratuitos ni consisten en una desviación respecto del significado propio: lo que cambia, cuando algo cambia, es la naturaleza del referente aludido, o el ámbito de uso, no el contenido semántico esencial” (p. 128).

Desde la concepción de estos estructuralistas, como se ve, se propone un análisis monosémico del contenido de las unidades léxicas en el nivel del sistema lingüístico -y consecuentemente en el diccionario-, puesto que "la multiplicidad de sentidos correspondería a una modulación contextual" (Rojas 2010: 16). Si bien esta perspectiva nos parece válida en algún sentido, y sobre todo desde la perspectiva de una metáfora sincrónica, aceptar

1 El concepto de metáfora sincrónica al que aquí se alude, refiere a la idea de que en la mente del hablante el significado de las voces es percibido como un haz, una red y no una serie de acepciones. Esta concepción se opone al modelo que reduce la relación entre acepción recta y figurada a una relación etimológica, donde el momento de creación metafórica se sitúa en un tiempo pretérito, y no ofrece posibilidad de recreación. 
de plano su validez nos impediría explicar los fenómenos históricos de transición semántica que generan el cambio lingüístico propiamente dicho, ahí cuando la metáfora deja de serlo y se genera realmente un nuevo sentido y ya no una extensión del primario, como señalara Coseriu (1977). En todo caso, desde la perspectiva de los modelos gramaticales generativistas y funcionalistas, cabría también refutar que el contorno no sea parte del significado implicado en una U.L., puesto que, en dichos modelos, es desde el lexicón o componente léxico desde donde se proyectan, hacia la sintaxis, las restricciones combinatorias de una unidad predicativa, por lo que, si bien no podemos determinar con precisión que se trate de rasgos contenidos dentro de lo que Pérez Castro llama "contenido semántico esencial", la naturaleza del referente y el ámbito de uso, sí son rasgos propios y determinantes de cada lexema.

Y de ahí el salto al otro extremo interpretativo de la relación que se establece entre acepciones: el paradigma generativista, que "considera que no existe relación entre unidades de contenido asociadas a la misma forma lingüística" (Rojas 2010:17), planteando que se puede tratar la multiplicidad de sentidos como homonimia. De más está decir que, en términos prácticos, este paradigma no ofrece soluciones lexicográficas viables. Pero además de ello, y pese a las interesantes pruebas de diferencialidad a las que Ignacio Bosque $^{2}$ (1982) ha sometido, por contraste, las acepciones figuradas y rectas de algunos ítems léxicos, tendemos a compartir la visión de una polisemia como una red semántica, puesto que percibimos que en el pensamiento analógico, lógico o metafórico, la concentración de la carga semántica, esto es, su capacidad de sintetizar y expresar contenidos, proviene justamente del cruce de campos conceptuales, tal como Lakoff (1980) lo describiera para sus estudios en relación con los conceptos metafóricos que proliferan en el lenguaje diario de lo que él ha llamado las metáforas de la vida cotidiana.

2 Bosque (1982) desarrolla una serie de pruebas con el fin de demostrar que las U.L. con sentido literal poseen un código léxico estructurado, aunque distinto de las con sentido literal u ordinario, por lo cual generan derivaciones morfológicas distintas, estructuras sintácticas distintas, oposiciones y relaciones semánticas de sinonimia distintas también. La competencia comunicacional de los hablantes, sin embargo, les permite codificar y decodificar correctamente estas secuencias que, si intentáramos comprender desde su sentido literal, nos parecerían agramaticales o inaceptables. Por ello, concluye que no solo tienen un código gramatical propio, sino que ofrecen un funcionamiento pragmático particular, cuyo estudio aún está pendiente y que será continuador, en algún sentido, de la tradición retórica clásica. 


\section{CONCLUSIONES}

Para no perder de vista el problema del tratamiento lexicográfico del sentido figurado, intentaremos revisar la solución del DRAE 2001, a la luz de los paradigmas recién expuestos: lo primero que podemos decir es que, al eliminar la marca fig., la Academia deconstruye, en apariencia, la dicotomía recto/figurado que había caracterizado su praxis hasta el diccionario de 1992. Lo segundo es que, pese a ello, al mantener la polisemia y no desintegrarla en homonimias monosémicas, da cuenta de una concepción que reconoce la existencia de una relación entre la primera acepción y las de sentido figurado y que, dado el orden en que se presentan dichas acepciones, establece también una jerarquía que posiblemente se estructure en torno a la oposición primario/ derivado y su implicación cronológica antes/después. Con ello, lo que se expresa es que la Academia aun mantiene una postura subsidiaria de la tradicional en lexicografía, al privilegiar un orden jerárquico de tipo histórico y no de frecuencia de uso, como ocurre teóricamente en el DEA. Por otra parte, la marca de estilo o registro coloq. estaría indicando al menos que se trata de usos especiales, aunque nada expresa ni sobre el origen ni sobre el sentido ni sobre la relación de esta acepción y las otras que componen la unidad polisémica. Cabe mencionar que DRAE 2001 tampoco marca las metonimias, el otro gran fenómeno de derivación semántica productor de nuevas acepciones. Por último, respecto de la fórmula "U.t.en sent.fig.", cabe destacar que parece dar cuenta de metáforas semigramaticalizadas, que bien pueden ameritar este tratamiento de subacepciones con comentario complementario.

Sin duda, el problema de cómo construimos significados está aún lejos de resolverse. Por ello, alcanzar el ideal de un diccionario de uso monolingüe, planteado por Lara, capaz de ser continente de la memoria semántica de una comunidad lingüística es, por ahora, una meta más que una ruta. Si pensamos en el artículo 'zorra' al que antes hemos referido, habría que aceptar que su cuarta acepción, que caracteriza a una persona astuta, establece una red semántica cultural que atraviesa no solo a la hembra del zorro, sino también a la personificación de ésta en tantas fábulas que son parte de nuestra memoria colectiva. Los estereotipos, las metáforas conceptuales, los mecanismos de adquisición del lenguaje nos permiten comenzar a acercarnos a la idea de que no comprendemos la realidad como parcelada, sino en redes de sentido, cuyas relaciones y jerarquías hemos intentado clasificar durante siglos.

Por ello, la lexicografía, cuya misión es la permanente reflexión y construcción de ese objeto diccionario, puede intentar sortear los límites físicos que le impone el objeto libro: linealidad, parcelación, limitación de 
espacio y remisión endofórica y buscar, tal vez aprovechando los nuevos soportes cuya metáfora principal es justamente la red, dar cuenta de las relaciones entre las U.L. desde donde, a nuestro entender, se genera el significado. Sin ir tan lejos, nos parece que los catálogos, que caracterizan la obra lexicográfica de Moliner, y los contornos, que caracterizan la de Seco, apuntan ya en esa dirección. Por otra parte, pero en este mismo sentido, las modas lexicográficas que apuntan a que la información etimológica e histórica de una voz deben quedar fuera del diccionario de uso nos parecen contradictorias, por cuanto, mientras el objetivo último del diccionario siga siendo el dar respuesta a la pregunta por el significado del léxico, estas informaciones aportarán información valiosísima en la construcción del mismo. La marca fig. en sí misma parece haberse mostrado incapaz de contener y expresar fenómenos tan complejos y productivos como la derivación semántica, por lo que su supresión parece acertada. Sin embargo, compartimos con Borràs y Torner (2006) que aún está pendiente la tarea de encontrar formas expresivas para el diccionario que den cuenta de la trama de relaciones desde la que los usuarios de una lengua construimos el sentido.

\section{REFERENCIAS BIBLIOGRÁFICAS}

Borràs, Laura y Sergi Torner. 2006. De nuevo sobre la marca figurado en los diccionarios. En Elisenda Bernal y Janet Decesaris (eds.). Palabra por palabra: estudios ofrecidos a Paz Battaner, pp. 33-45. Barcelona: Institut Universitari de Lingüística Aplicada, Universitat Pompeu Fabra.

Bosque, Ignacio. 1982. Más allá de la lexicalización. Boletin de la Real Academia Española, Tomo 62, Cuaderno 225: 103-158.

Coseriu, Eugenio. 1977. Principios de semántica estructural. Madrid: Gredos.

DE Hoyos, José Carlos. 1999-2000. La marca de transición semántica: sentido figurado. Revista de lexicografia VI: 73-105.

DEA. 1999. Seco, Manuel et Al. Diccionario del español actual. Madrid: Aguilar.

DRAE. 1970. Real Academia Española. Diccionario de la Lengua Española. Madrid: Espasa Calpe (19a ed.).

DRAE. 1992. ReAl Academia Española. Diccionario de la Lengua Española. Madrid: Espasa Calpe ( $21^{\mathrm{a}}$ ed.).

DRAE. 2001. Real Academia Española. Diccionario de la Lengua Española. Madrid: Espasa Calpe (22 ed.).

DUE. 1999. Moliner, María. Diccionario de uso del español. Madrid: Gredos.

FAJARDO, AlejANDRO. 1996-1997. Las marcas lexicográficas: concepto y aplicación práctica en la Lexicografía española. Revista de Lexicografía 111: 31-57.

Lakoff, George y Mark Johnson. 1980. Metáforas de la vida cotidiana. Madrid: Ediciones Cátedra. 
Lara, Luis Fernando. 1990. El objeto diccionario. En Luis Fernando Lara. Dimensiones de la lexicografía: A propósito del Diccionario del español actual de México, pp. 21-38. México: El Colegio de México.

Pérez Castro, Luis Carlos. 2002. Acerca de los usos y sentidos figurados y de su tratamiento lexicográfico. Revista española de lingüistica año 32, 1: 123-128.

ROJAS, DARÍo. 2010. El tratamiento de la polisemia léxica regular y las facetas en una muestra de diccionarios monolingües de español. Tesis para optar al grado de Magíster en Lingüística con mención Lengua Española, Universidad de Chile. [En línea] Disponible en http://www. cybertesis.uchile.cl/tesis/uchile/2010/fi-rojas_da/html/index.html [Consulta 28/03/2011]

Seco, Manuel. 1978. Problemas formales de la definición. En Manuel Seco. Estudios de lexicografía española, pp.15-34. Madrid: Paraninfo. 